\title{
Improved Energy Efficient Routing Wireless Sensor Networks Based On Clusters
}

\author{
Gobu .N.K ${ }^{1}$, Dr.S.Prema ${ }^{2}$ \\ M. Phil Research Scholar, Department of Computer Science (PG), \\ K.S.Rangasamy College of Arts and Science (Autonomous), Tiruchengode, Tamilnadu, India ${ }^{1}$ \\ Associate Professor, Department of Computer Science (PG), \\ K.S.Rangasamy College of Arts and Science (Autonomous), Tiruchengode, Tamilnadu, India ${ }^{2}$
}

\begin{abstract}
Wireless sensor networks are sparsely connected sovereign gadgets, which are incorporated with the sensors. They are deployed in amidst of the monitoring of physical environments. WSN system makes and interconnection between the wired and wireless nodes. A sensor node incorporates the ambient, temperature, humidity and pedometer aspects whichever can be incorporated based the circumstances. Higher number of signals involved needs proper routing channels to achieve successful communication.Routing is a process of divide and conquer technique, which initiates in transferring information through various routes and receiving packets at destination and reassemble them in order to achieve better communication. This research proposes an algorithm, which focus on proficient energy handling in routing of the WSN sensors. The proposed algorithm focuses on the dynamic cluster head selection and proficient handling of the energy.
\end{abstract}

Keywords: Wireless Sensor Network, Weight Based Clustering Algorithms (WCA)

\section{INTRODUCTION}

Wireless sensor networks are connected with gadgets, which are unified with the sensors. They are positioned in amidst of the observing of physical atmospheres. WSN system makes and interconnection amongst the wired and wireless nodes. Sensor nodes integrates the ambient, temperature, humidity and pedometer aspects either can be incorporated based the circumstances. Sophisticated number of gestures involved needs apt routing channels to achieve successful communication.Routing is a process of divide and conquer technique, which initiates in transferring information through various routes and receiving packets at destination and reassemble them in order to achieve better communication. This research proposes an algorithm, which focus on remarkable energy handling in routing of the WSN sensors. The proposed algorithm focuses on the dynamic cluster head selection and proficient handling of the energy. Energy consumption which shows the proposed algorithm performs the best and the base works which has been considered for the comparison are weight based clustering algorithms (WCA) by Chatterjee et al. and Improved WCA by the same author.This exploration intends an algorithm, which focus on dexterous energy handling in routing of the WSN sensors. The proposed algorithm focuses on the dynamic cluster head selection and proficient handling of the energy.Results are promising and would improve the performance of the network lifetime.

\section{LITERATURE REVIEW}

A. An Efficient Genetic Algorithm for Anycast Routing in Delay/Disruption Tolerant Networks

R.Silva and Paulo et.a1 [1] Delay/Disruption tolerant networks (DTNs) have the capacity to interconnect devices and areas that are underserved by traditional networks. Any cast routing can be used for many applications in DTNs, and is useful when nodes wish to send messages to at least one, and preferably only one, of the members in a destination group. The proposed GA-based solution uses the concept of subpopulation to produce the next generation population.

B. Analytical Query Response Time Evaluation for a Two-Level Clustering Hierarchy Based Wireless Sensor Network Routing Protocol

Siva D. Muruganathan et.a1 [2] we analytically evaluate the average query response time of the Two-level Hierarchical Clustering based Hybrid-routing Protocol (THCHP) proposed recently for wireless sensor networks (WSNs). The accuracy of the average query response time analysis is verified via numerical simulations. Additionally, average query response time comparisons.

C. Efficient Algorithms for Topology Control Problem with Routing Cost Constraints in Wireless Networks 


\section{International Advanced Research Journal in Science, Engineering and Technology}

Vol. 5, Issue 11, November 2018

Ling Ding et.a1 [3] a wireless network is an especially important factor to influence the performance of the network in terms of broadcasting, scheduling of transmission, and routing Thus, many researchers focus on removing such kind of useless topology information from a network which is called topology control or topology management. It is believed that topology control can dramatically improve a network's broadband utilization and delivery ratio, extend network lifetime, and reduce interference.

D. Assessment of the Power Series Routing Algorithm in Translucent, Transparent and Opaque Optical Networks Joaquim F. Martins-Filho et.a1 [4] in this letter we extend the analysis of the Power Series Routing (PSR) algorithm, which was previously proposed and analyzed for transparent networks only, to the translucent and opaque networks. The PSR algorithm is a bio-inspired impairment-aware routing algorithm that can be optimized and can adapt itself to the network conditions. We demonstrate that the PSR algorithm is also suitable for these network configurations.

E. MAP: MulticonstrainedAnypathRouting in Wireless Mesh Networks

Xi Fang, Dejun Yang et.a1 [5] routing algorithms and protocols for wireless networks often follow the design methodology for wired networks by abstracting the wireless links as wired links and looking for the shortest delay, least cost, or widest bandwidth path(s) between a pair of source and destination nodes. However, for unreliable wireless networks, due to the broadcast nature of the wireless medium, it is usually less costly to transmit a packet to one of the nodes in a set of neighbors than to one specific neighbor.

F. An Oblivious Routing Algorithm for 3D Mesh Networks to Achieve a New Worst-Case Throughput Bound Guang Sun, Chia-Wei Chang et.a1 [6] 1/2 network capacity is often believed to be the limit of worst-case throughput for three-dimension (3D) mesh networks. However, this paper provides a new worst-case throughput bound, which is higher than 1/2 network capacity, for odd radix 3D mesh networks. In addition, we propose a routing algorithm called uniform solo-minimal (USM) routing that can achieve this new worst-case throughput bound in odd radix mesh networks. For the even radix case, we prove that USM achieves the optimal worst-case throughput, namely, half of network capacity. We proposed a new routing algorithm for 3Dmesh called USM that achieves our new worst-case throughput bound when the radix is odd and optimal worst-case throughput when the radix is even.

\section{IDENTIFIED PROBLEM FROM EXISTING SYSTEM}

A wireless sensor network (WSN) (or IoT network) is a collection of distributed nodes. These nodes gather data from various sensors and relay that information to a central point through a wireless network. Therefore, the data can be aggregated and have something useful done with it. These types of networks deal primarily with the transmission of small amounts of data that needs to be sent very efficiently. In this paper, we propose an energy-efficient routing algorithm based on the global positioning system (GPS) information. Proposed scheme track the destination's location based on the beacon messages of the main route nodes.

\section{PROPOSED WORK: WIRELESS SENSOR NETWORKS BASED ON CLUSTERS}

Energy consumption which shows the proposed algorithm performs the best and the base works which has been considered for the comparison are weight based clustering algorithms (WCA) by Chatterjee et al. and Improved WCA by the same author.This research proposes an algorithm, which focus on proficient energy handling in routing of the WSN sensors. The proposed algorithm focuses on the dynamic cluster head selection and proficient handling of the energy. Results are promising and would improve the performance of the network lifetime.

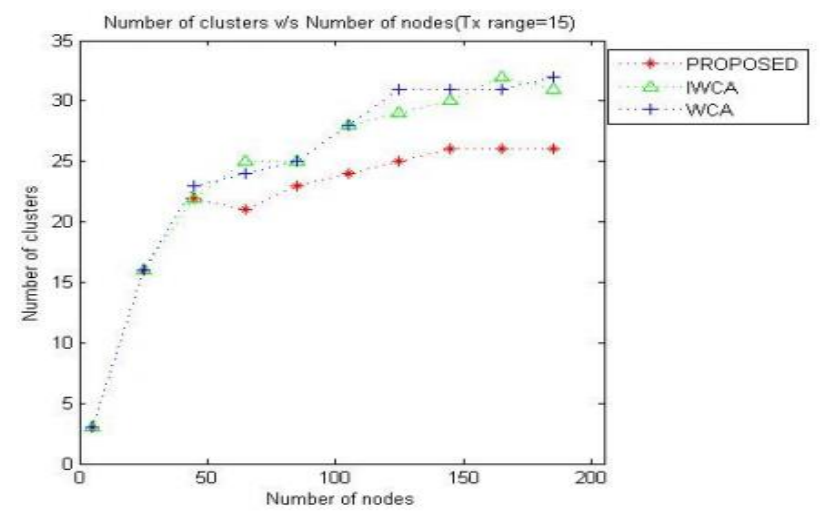

Figure 1: Clusters Number and Total Number of Nodes 


\section{International Advanced Research Journal in Science, Engineering and Technology}

Vol. 5, Issue 11, November 2018

Energy consumption which shows the proposed algorithm performs the best and the base works which has been considered for the comparison are weight based clustering algorithms (WCA) by Chatterjee et al. and improved WCA by the same author.This research proposes an algorithm, which focus on proficient energy handling in routing of the WSN sensors. The proposed algorithm focuses on the dynamic cluster head selection and proficient handling of the energy. Results are promising and would improve the performance of the network lifetime.

- Preliminary phase: To begin with, the algorithm starts its working with dispersing the nodes of the sensors and followed by coverage of the area within the predicted area. Later the number of hops will be recorded which may be permitted up to 2 . Then the broadcast details will be recorded as well with the cluster head and nodes.

- Grouping phase: The grouping of the sensor nodes will be done first. Followed by cluster lead formation. Now the list will be broadcasted to all the cluster head and maintained.

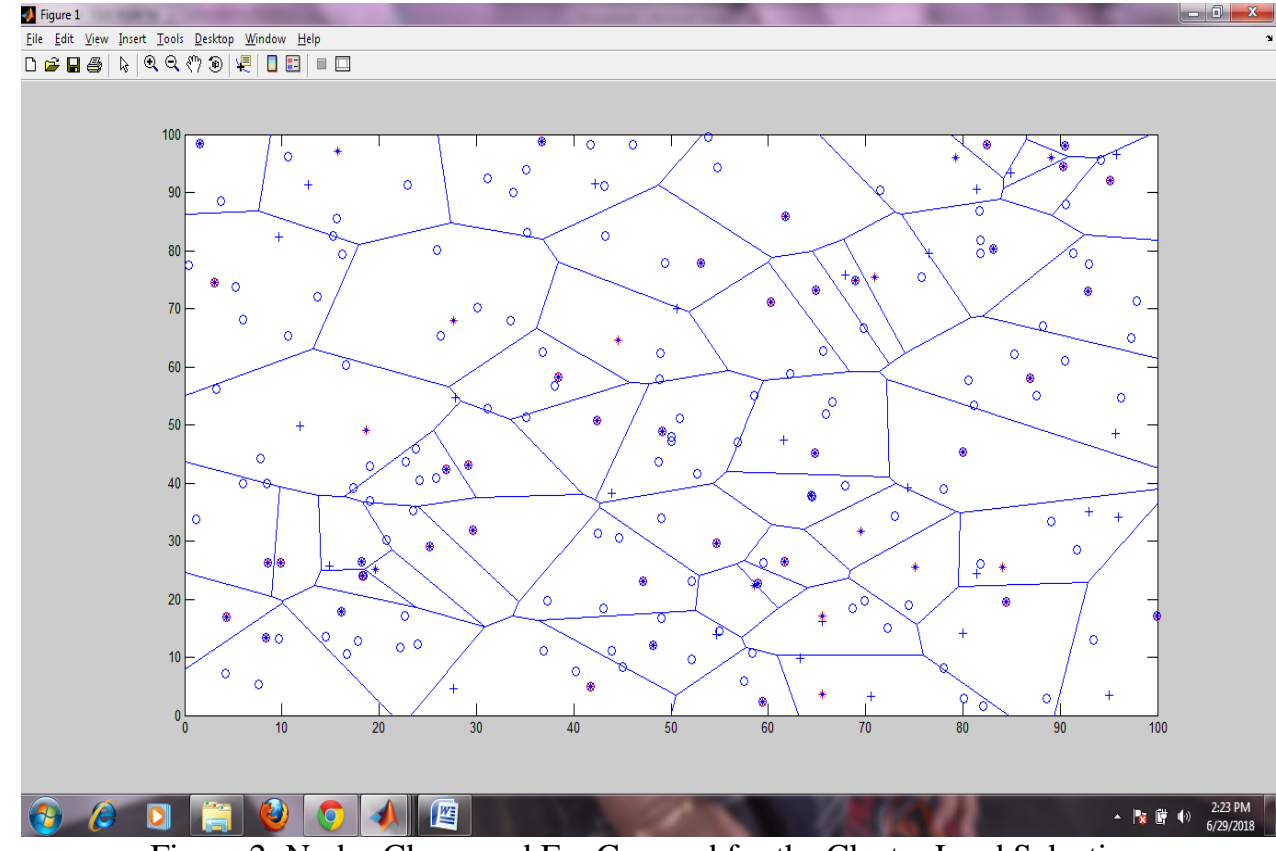

Figure 2: Nodes Closer and Far Grouped for the Cluster Lead Selection

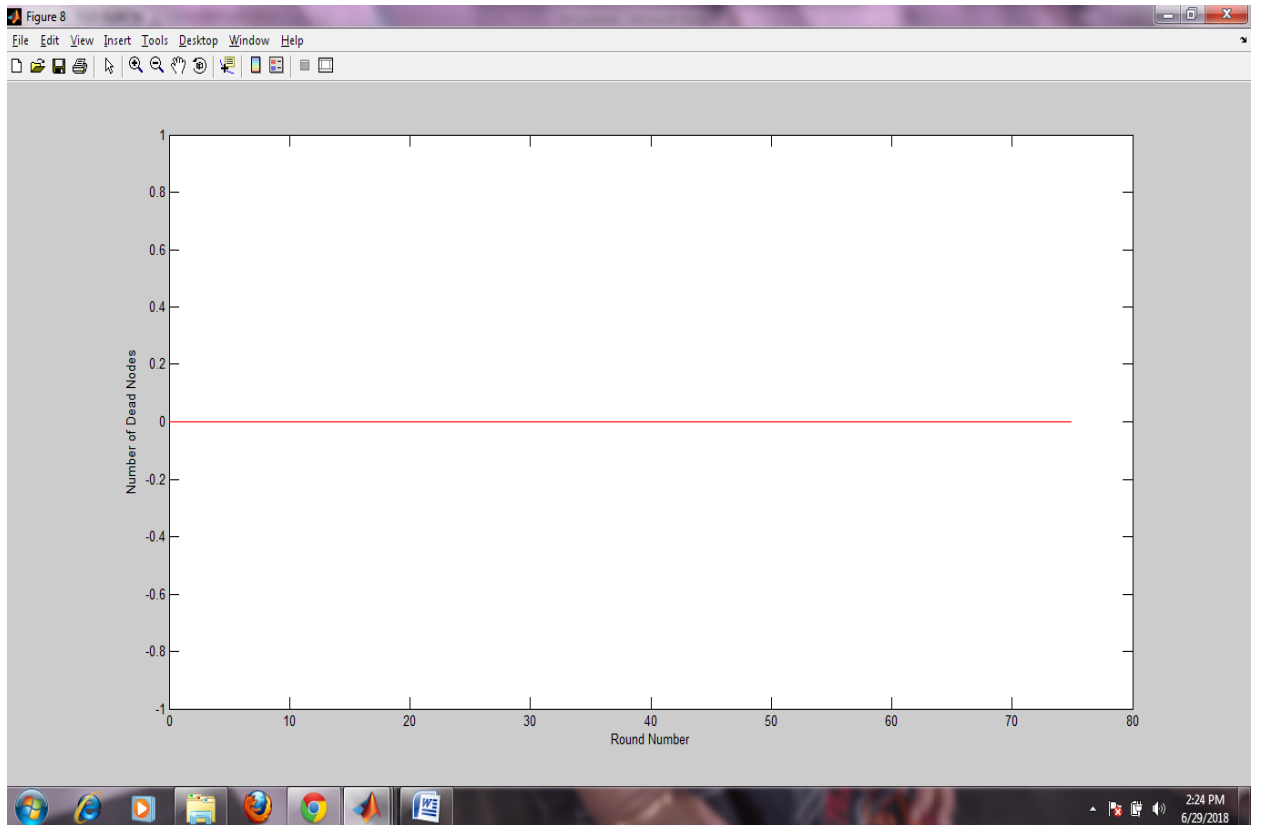

Figure 3: Number of Dead Nodes after Stability

\section{CONCLUSION}




\section{International Advanced Research Journal in Science, Engineering and Technology}

Vol. 5, Issue 11, November 2018

Wireless sensor networks are sparsely allied sovereign gadgets, which are unified with the sensors. They are positioned in amidst of the observing of physical atmospheres. WSN system makes and interconnection amongst the wired and wireless nodes. Sensor nodes integrates the ambient, temperature, humidity and pedometer aspects either can be incorporated based the circumstances. Sophisticated number of gestures involved needs apt routing channels to achieve successful communication.

\section{CONTRIBUTIONS TO THE KNOWLEDGE}

Routing is a process of divide and conquer technique, which initiates in transferring information through various routes and receiving packets at destination and reassemble them in order to achieve better communication. This exploration intends an algorithm, which focus on dexterous energy handling in routing of the WSN sensors. The proposed algorithm focuses on the dynamic cluster head selection and proficient handling of the energy. Results are promising and would improve the performance of the network lifetime.Energy consumption which shows the proposed algorithm performs the best and the base works which has been considered for the comparison are weight based clustering algorithms (WCA) by Chatterjee et al. and Improved WCA by the same author.This research proposes an algorithm, which focus on proficient energy handling in routing of the WSN sensors. The proposed algorithm focuses on the dynamic cluster head selection and proficient handling of the energy. Results are promising and would improve the performance of the network lifetime. It is concluded that the proposed algorithm works efficiently.

\section{FUTURE WORK}

Dynamic path finding and routing could be enhanced further with the help of the adaptive algorithms such as genetic algorithm and self-organizing maps. Energy drain is a major problem in communication. When the cluster heads seems to be having the energy drain it will lead to node death and consequently, if cluster head dies the entire information gathered would be lost. Focusing on the network dynamics and adaptability in reframing the cluster heads will also help in the preventing the network information loss. These are the further enhancements for the proposed research.

\section{REFERENCES}

[1]. Ederson r. silva and paulo r. guardieiro "An efficient genetic algorithm for any cast routing in delay/disruption tolerant networks" an efficient genetic algorithm for any cast routing in delay/disruption tolerant networks Vol. 14, No. 4, April 2010, IEEE.

[2]. Siva d. muruganathan "Analytical query response time evaluation for a two-level clustering Hierarchy based wireless sensor network routing protocol" analytical query response time evaluation for a two-level clustering hierarchy based wireless Vol. 14, No. 5, May 2010, IEEE.

[3]. Ling Ding "Efficient Algorithms for Topology Control Problem with Routing Cost Constraints in Wireless Networks" Connected Dominating Set (CDS) Vol. 22, No.10, October 2011, IEEE.

[4]. Joaquim F. Martins-Filho "Assessment of the Power Series Routing Algorithm inTranslucent, Transparent and Opaque Optical Networks" Vol. 16, No. 6, June 2012, IEEE.

[5]. Xi Fang "Map: MulticonstrainedAnypath Routing in Wireless Mesh Networks"Transactions On Mobile Computing, Vol. 12, No. 10, October 2013, IEEE.

[6]. Guang Sun, Chia-Wei Chang "An Oblivious Routing Algorithm For 3d Mesh Networks To Achieve A New Worst-Case Throughput Bound" Vol. 4, No. 4, December 2012, IEEE.

\section{BIOGRAPHIES}

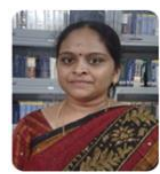

Dr.S.Prema, currently working as an Associate Professor in K.S.Rangasamy College of Arts \& Science has received Ph.D., from the Bharathiar University in 2015. She has been involved in the teaching for the past 13 years. She secured the $1^{\text {st }}$ Rank in B.Sc under Periyar University, Salem. She has totally 52 publications and one of her research paper entitled "An NLP based Approach for Facilitating Efficient Web Search Results using BSDS"received the best paper award. Her papers are cited at various publications (IEEE Xplore, Elsevier, Springer and International Conference Proceedings). She has h-index value: 5, i10 index: 3, Citations: 125 and her profile is listed in Marquis Who is Who in World, International Biography Center London, UK, 2011. She has been awarded "Innovative Research \& Dedicated Women Academician Award" at International Awards \& Honors Convocation 2018 Conducted by The Society of Innovative Educationalist \& Scientific Research Professional, Malaysia. She has produced 5 M.Phil scholars and currently guiding 4 M.Phil and 1 Ph.D Scholars for doing their research. She has been involved in generating funds for R\&D.

Mr. Gobu N.K is pursuing M.Phil (Computer Science) in K.S.Rangasamy College of Arts and Science (Autonomous), Tamilnadu, India. He has completed M.SC (CS) degree under Periyar University, in 2016. He has attended 4 workshops, 3 seminar related to Network and presented a paper related to his research work in an International conference. His areas of interest are Network and Data Mining. 